\title{
Household food insecurity and childhood overweight in Jamaica and Québec: a gender-based analysis
}

\author{
Lise Dubois $^{1{ }^{*}+}$, Damion Francis ${ }^{2 \dagger}$, Daniel Burnier ${ }^{3 \dagger}$, Fabiola Tatone-Tokuda ${ }^{3 \dagger}$, Manon Girard ${ }^{3 \dagger}$, \\ Georgiana Gordon-Strachan ${ }^{4 \dagger}$, Kristin Fox ${ }^{5+}$ and Rainford Wilks ${ }^{2+}$
}

\begin{abstract}
Background: Childhood overweight is not restricted to developed countries: a number of lower- and middleincome countries are struggling with the double burden of underweight and overweight. Another public health problem that concerns both developing and, to a lesser extent, developed countries is food insecurity. This study presents a comparative gender-based analysis of the association between household food insecurity and overweight among 10-to-11-year-old children living in the Canadian province of Québec and in the country of Jamaica.

Methods: Analyses were performed using data from the 2008 round of the Québec Longitudinal Study of Child Development and the Jamaica Youth Risk and Resiliency Behaviour Survey of 2007. Cross-sectional data were obtained from 1190 10-year old children in Québec and 1674 10-11-year-old children in Jamaica. Body mass index was derived using anthropometric measurements and overweight was defined using Cole's age- and sex-specific criteria. Questionnaires were used to collect data on food insecurity. The associations were examined using chi-square tests and multivariate regression models were used to estimate odds ratios $(O R)$ and $95 \%$ confidence intervals.

Results: The prevalence of overweight was $26 \%$ and $11 \%(p<0.001)$ in the Québec and Jamaican samples, respectively. In Québec, the adjusted odds ratio for being overweight was 3.03 ( $95 \%$ Cl: 1.8-5.0) among children living in food-insecure households, in comparison to children living in food-secure households. Furthermore, girls who lived in food-insecure households had odds of 4.99 (95\% Cl: 2.4-10.5) for being overweight in comparison to girls who lived in food-secure households; no such differences were observed among boys. In Jamaica, children who lived in food-insecure households had significantly lower odds (OR 0.65, 95\% Cl: 0.4-0.9) for being overweight in comparison to children living in food-secure households. No gender differences were observed in the relationship between food-insecurity and overweight/obesity among Jamaican children.
\end{abstract}

Conclusions: Public health interventions which aim to stem the epidemic of overweight/obesity should consider gender differences and other family factors associated with overweight/obesity in both developed and developing countries.

\section{Background}

In most countries where data are available, the prevalence of childhood overweight has increased [1] to the point of becoming a major public health problem. Although there is some indication that this epidemic may be leveling-off in certain countries over recent years, this

\footnotetext{
* Correspondence: Idubois@uottawa.ca

+ Contributed equally

'Faculty of Medicine, University of Ottawa, Institute of Population Health, 1

Stewart Street, office 303, Ottawa, Ontario, K1N 6N5, Canada

Full list of author information is available at the end of the article
}

evidence is less apparent in lower-SES groups and does not seem to be the case for Canada and some European and Asian countries [2]. Childhood overweight is also associated with numerous short and long-term physiological and psychosocial negative health consequences in both individuals and populations [3-6]. Some studies purport that the epidemic of overweight is due to an increased consumption of low-nutrient, energydense products that are high in sugar and fats [7-11].

\section{Biomed Central}

(c) 2011 Dubois et al; licensee BioMed Central Ltd. This is an Open Access article distributed under the terms of the Creative Commons Attribution License (http://creativecommons.org/licenses/by/2.0), which permits unrestricted use, distribution, and reproduction in any medium, provided the original work is properly cited. 
Others suggest that weight increases are mainly attributable to physical inactivity $[10,11]$.

The childhood overweight epidemic is not restricted to developed countries: a number of lower- and middleincome countries have been struggling with the double burden of underweight and overweight for some time $[12,13]$. In urban areas of countries undergoing rapid social and economic change (e.g., China, Mexico, Egypt, and Brazil), the prevalence of overweight among children has reached levels comparable to those in developed countries [14]. In western developed countries, childhood adiposity has, for the most part, been shown to inversely associate with socioeconomic status (SES), according to a systematic review of studies from 19902005 [15]; whereas, in developing countries, a greater prevalence of childhood overweight has been observed in higher socioeconomic groups [1]. Some gender differences in this association have been noted, however. A seminal review of 144 published studies on the association between SES and overweight/obesity found a strong inverse relationship particularly among women in developed societies; this relationship was inconsistent for men and children. In developing countries, however, a direct association was observed between SES and obesity among men, women, and children [16,17], although a high prevalence of obesity has been reported even among very poor women in developing countries [18].

Another public health problem that concerns both developing and, to a lesser extent, developed countries is food insecurity. Food insecurity arises when individuals do not have sufficient access to safe and nutritious foods at all times, to sustain active and healthy lives [19]. Some studies have reported a paradoxical positive association between food insecurity and childhood obesity [20-22]. However, there have been some inconsistent findings in this research area, with some studies reporting a negative association [23-25] or completely non-existent association between food insecurity and childhood obesity [26,27].

Thus, this study aims, firstly, to examine whether household food insecurity is significantly related to child overweight/obesity in the Canadian province of Québec (total population of less than 8 million) and in the country of Jamaica (total population less than 3 million) and, secondly, to explore gender differences in the association between food insecurity and overweight/obesity in both polities. This study is part of an ongoing collaboration between the University of the West Indies' Epidemiology Research Unit (based in Kingston, Jamaica) and the Institute of Population Health (University of Ottawa, Canada). The prevalence of childhood overweight/obesity is high in both Canada and Jamaica: $26 \%$ of 6 -to- 11 year-old Canadian children were overweight or obese in 2004 according to the age- and sex-specific criteria developed by the International Obesity Task Force [28]; while, in Jamaica, the prevalence of overweight in 11-to12 -year-old children living in the Kingston Metropolitan area was reported to be $19 \%$ (BMI $\geq 85^{\text {th }}$ percentile) in 1998 [29]. Furthermore, data from the Canadian Community Health Survey revealed that approximately $7 \%$ of Québec households were food-insecure in 2007-2008 [30]. Data on the prevalence of food insecurity among Jamaican households, however, has yet to be published. For the present study, it was hypothesized that a positive association would be observed between household food insecurity and childhood overweight/obesity in Québec, while a negative association would be observed in Jamaica, independent from other factors potentially associated with child overweight/obesity.

\section{Methods \\ Background on study samples \\ Québec}

Analyses were conducted using data from the Québec Longitudinal Study of Child Development (QLSCD), a study conducted by Santé Québec, a division of the Institut de la Statistique du Québec (ISQ) [31]. Approval from the Ministry of Health Ethics Committee and consent from participants were obtained. The QLSCD, established to examine the role of familial and social factors in children's health, cognitive, and behavioural development, followed a representative sample $(\mathrm{n}=$ 2103) of children born in 1998, in the province of Québec (approximately 70,000 newborns per year), Canada. To ensure geographic representation and minimize the effect of seasonality, the study randomly selected children born throughout the year in each public health geographic area of the province. A public health geographic area or "health region" refers to a geographic unit defined by the provincial ministry of health. Health regions facilitate public health administration for Canadians. Children and their parents were first seen at 5 months (gestational age adjusted for preterm birth) and at one-year intervals thereafter. Standardized, questionnaire-based face-to-face interviews and self-administered questionnaires, completed by the children's mothers and fathers, were used at each cycle of data-collection. Data pertaining to the child was obtained from the person deemed most knowledgeable about the child, which generally was the mother. Information was also obtained from the child's medical records. Of the 2103 infants included in the first cycle of the study, 1190 children remained in the study 10 years later, in 2008.

\section{Jamaica}

Jamaican data were drawn from the Jamaica Youth Risk and Resiliency Behaviour Survey 2005, conducted by the University of the West Indies [32]. The main purpose of this cross-sectional survey was to monitor the health 
status, nutritional habits, and lifestyles of children and young teenagers aged 10-15 years, in a nationally representative sample of Jamaican children currently enrolled in school, and to examine how these variables relate to demographic and socioeconomic factors. The data were obtained from the children using trained interviewers who administered questionnaires which were standardized and validated for use in this population. Most children attended primary or secondary schools regularly. The average daily rate of attendance for primary school children was 78.5\% [33,34]. Enrolment records, obtained from the Ministry of Education, Youth and Culture, and school attendance registers from selected schools provided the sample frames used in this study.

A multi-stage random sampling method was employed. The first stage involved the random selection of schools within each region where probability was proportional to size. The second stage entailed randomly selecting children from grades within the required age groups. The number of schools selected and the number of students selected per school were proportional to the total number of children in the required age group per parish and school. In 2003, there were 279,986 children in the 10-to-14-year age group and 250,352 in the 15to-19-year age group. These combined represented approximately $20 \%$ of the Jamaican population [35]. Using information on the rate of tobacco use among youths aged 10 to 14 (19\%) [36], a confidence level of $95 \%$ and an error of $+2 \%$ yielded a required sample size of 2,500 children (EPI-Info software obtained from the Centers for Disease Control and Prevention [CDC] website). Based on an expected refusal rate of $10 \%$, the sample size was adjusted to 2,800. For this analysis, 1674 children between ages 10 and 11 years were selected for study.

\section{Measures}

\section{Overweight and obesity}

In Québec, children's heights and weights were measured at home by a trained interviewer following a standardized protocol using a measuring tape, ruler, and scale [37] when children were 10 years old. In Jamaica, body weight, without shoes and with light clothing, was recorded to the nearest $0.1 \mathrm{~kg}$ using a calibrated electronic platform scale. Standing height was recorded to the nearest $0.1 \mathrm{~cm}$ using a Leicester portable measuring rod. Measurements were obtained at school by trained interviewers following a standardized study protocol. Body mass index (BMI) was calculated as body weight $/$ height $^{2}$ $\left(\mathrm{kg} / \mathrm{m}^{2}\right)$. Overweight and obesity were similarly defined in Québec and Jamaica according to Cole's criteria, which provide age- and sex-specific cut-off points for overweight and obesity in children between 2 and 18 years of age [38].

\section{Food insecurity}

Food insecurity was assessed in comparable ways in Québec and Jamaica, and fit within the definitional ambits adopted by both the Food and Agriculture Organization (FAO) and the United States Department of Agriculture (USDA) $[19,39,40]$. In the Québec sample, data on food insecurity were collected via self-administered questionnaires addressed to mothers when children were 10 years of age. Using a 3-point Likert-type scale (rated "Often true", "Sometimes true", "Never true"), mothers were asked to rate how often their families had experienced each of the three following situations: 1) We eat the same thing several days in a row because we only have a few different kinds of food on hand, and don't have enough money to buy more; 2) We eat less than we should because we don't have enough money for food; and 3) We can't provide balanced meals for our children because we can't afford it financially. Children were classified as living in a food-insecure household if mothers answered "often true" or "sometimes true" to any of the three food insecurity statements, and as living in a food-secure household if mothers answered "never true" to each statement.

In the Jamaican sample, children were interviewed to ascertain the presence or absence of food insecurity as well as the extent to which they were food-insecure using two structured questions. The first dichotomous question was: 1) During a usual week, do you go hungry because there is not enough food in your home? (rated "Yes" or "No"). Children were categorized as being foodinsecure if they answered yes to question 1 . They were then asked how often they experienced hunger using a 4-point Likert-type rating scale to categorize the extent of food insecurity: 2) During a usual week, how often do you go hungry because there was not enough food in your home? (rated "Always", "Most of the time", "Sometimes", "Rarely"). This method of combining responses has been employed in other studies on food-insecurity $[41,42]$. In both questionnaires, the food-insecurity statements gather information at the household level, which has been demonstrated to have both face validity $[43,44]$ and external validity $[45,46]$.

\section{Diet and physical activity}

In Québec, food consumption (daily consumption of pastries, fruits, and vegetables) was measured by way of a self-administered Food Frequency Questionnaire (FFQ) completed by the children's mothers when the children were 10 years old. The children's mothers were asked: In the past week, at home and at school (or school's daycare service), on average, how many times during the week or how many times per day has your child eaten the following foods. The mothers chose one of the following responses: "none", "one to two times per week", "three to four times per week", "five to six 
times per week", "one time per day", "twice per day", "three times per day", or "four or more times per day". Daily consumption of pastries included pastries, candies, cookies, chips, and chewing gum containing sugar. Daily consumption of fruits excluded the consumption of fruit drinks or juice. Vegetable consumption included potatoes. Parents reported children's level of physical activity by stating whether their child had a "higher" or "much higher" level of physical activity in comparison to other children, or "same", "lower" or "much lower" level than other children. Children's level of physical activity was reported by the parents at age 6 . All other variables included in the present study were completed when the children were 10 years old.

In Jamaica, children provided information about their dietary consumption patterns throughout a usual week (i.e., a week without social events that might affect usual intake). A standard questionnaire was validated for use in similar populations of adolescents [47]. Two questions were asked specifically about fruit consumption: (1) During a usual week, do you eat fruit such as mango, orange, and pawpaw? Response categories for this first question were: "yes", "no" and "don't know". If the child's response was "yes", then the interviewer proceeded to the following question: (2) During a usual week, how many times per week do you usually eat fruit, such as mango, orange, and pawpaw? Response categories were " $<1,1,2,3,4,5$ or more, and don't know". A similarly structured set of questions was asked about usual weekly and daily consumption of vegetables (which did not include potatoes) and pastries (cake, bulla cake, buns, etc.). Physical activity was assessed using the short form of the International Physical Activity Questionnaire (IPAQ) [48] for leisure time physical activity, and children's level of physical activity was classified as "none or low" or "moderate or high".

\section{Family type and socioeconomic status (SES)}

In Québec, the SES index was calculated by the Institut de la Statistique du Québec [49] based on methods developed by Willms and Shields (1996) that aggregate annual gross income, education level, and occupational prestige for both parents [50]. SES was less rigorously defined in the Jamaican sample due to the unavailability of data: it was based on household crowding terciles and a geographical index (urban, rural, remote rural) developed by the Statistical Institute of Jamaica (STATIN). These two variables were combined to derive a proxy for SES. Family type categories were two-parent family, blended family, and single-parent family in both the Québec and Jamaican samples.

\section{Statistical analyses}

Statistical analyses were conducted using SAS (version 9.2; SAS Institute; Cary, NC) and Stata (Version 10.1
Stata Corp.). In Québec, data were weighted by a factor based on the inverse of the selection probability, the probability of non-response, and the post-stratification and attrition rates to ensure that the data were longitudinally representative of infants born in 1998, in the province of Québec [51]. The weights used were provided by the Institut de la Statistique du Québec and included corrections for response bias. Thus, the rates obtained through this study are comparable to those from other surveys on the same population that use weighted responses, independent of the distribution of the sample [37]. The Jamaican data were weighted by age, sex, school type, and parish to provide normalized weights using data from STATIN.

Univariate associations were verified by performing a chi-square test on contingency tables. The significance level was set at 5\%, and significant independent variables and other putative explanatory variables were included in multivariate analyses. Based on the literature, children's sex, level of physical activity, family type, family SES (by tertile: low, medium, high), and children's daily consumption of fruit, vegetables, and pastries were considered potential confounders. Our final models included food insecurity, SES, and family type.

In the Québec model, adjustments were made for pastry and vegetable consumption (significantly associated in the univariate analysis) and physical activity (due to its integral role in the equation of energy balance). The Jamaican model was adjusted for physical activity (significantly associated in the univariate analysis). Kendall Tau's test for correlation was also employed to ensure that SES and household food insecurity were not highly correlated and could be included in the same model as independent variables. Odds ratio (OR) estimates, with 95\% confidence intervals, were computed using logistic regressions. The impact of missing data was assessed by conducting with-and-without analyses, which revealed no effect on the outcomes and results seen.

\section{Results}

Characteristics of the Québec and Jamaican children are presented in Table 1. Approximately one-quarter (26\%) of children from Québec and one-tenth (11\%) of children from Jamaica were overweight or obese. Food insecurity was reported for 9\% of the children in Québec and $26 \%$ in Jamaica. A greater proportion of children lived in single-parent families in Jamaica (36\%) in comparison to Québec (17\%). Fruit and vegetable consumption was higher in Québec than in Jamaica, whereas the difference in physical activity was less marked.

Table 2 presents the prevalence of childhood overweight/obesity in Québec and Jamaica according to selected child, family, and household characteristics. In Québec, when boys and girls were analyzed together, 
Table 1 A comparison of 10-year-old Québec and 10-11-year-old Jamaican study participants

\begin{tabular}{|c|c|c|c|c|}
\hline & & Québec & Jamaica & \\
\hline Characteristic & Category & $\%(n)$ & $\%(n)$ & P-value \\
\hline \multirow[t]{2}{*}{ Sex } & Girl & $52.4(624)$ & $52.6(881)$ & 0.6792 \\
\hline & Boy & $47.6(566)$ & $47.4(793)$ & \\
\hline \multirow[t]{2}{*}{ Overweight or obese } & No & 74 & 89 & $<.0001$ \\
\hline & Yes & 26 & 11 & \\
\hline \multirow[t]{3}{*}{ Family SES } & Tertile 1 (Low) & 33.0 & 41.3 & $<.0001$ \\
\hline & Tertile 2 (Middle) & 34.1 & 29.4 & \\
\hline & Tertile 3 (High) & 32.9 & 29.3 & \\
\hline \multirow[t]{2}{*}{ Household food insecurity } & No & 90.6 & 73.6 & $<.0001$ \\
\hline & Yes & 9.4 & 26.4 & \\
\hline \multirow[t]{3}{*}{ Family type } & Two-parent family & 66.3 & 34.2 & $<.0001$ \\
\hline & Blended family & 17.0 & 29.7 & \\
\hline & Single-parent family & 16.7 & 36.1 & \\
\hline \multirow[t]{3}{*}{ Pastry consumption } & Less than once a day & 68.7 & 64.2 & 0.1375 \\
\hline & Once to less than twice a day & 20.3 & 23.4 & \\
\hline & Twice a day or more & 11.0 & 12.4 & \\
\hline \multirow[t]{3}{*}{ Fruit consumption } & Less than once a day & 31.1 & 41.5 & $<.0001$ \\
\hline & Once to less than twice a day & 19.1 & 31.9 & \\
\hline & Twice a day or more & 49.8 & 26.6 & \\
\hline \multirow[t]{3}{*}{ Vegetable consumption } & Less than once a day & 27.1 & 59.0 & $<.0001$ \\
\hline & Once to less than twice a day & 18.8 & 25.3 & \\
\hline & Twice a day or more & 57.1 & 15.7 & \\
\hline \multirow[t]{2}{*}{ Physical activity (Québec) } & Same, lower or much lower than other children & 67.6 & $/ / / / / / / / / /$ & \\
\hline & Higher or much higher than other children & 32.4 & ////////// & \\
\hline \multirow[t]{2}{*}{ Physical activity (Jamaica) } & None or low & $/ / / / / / / / /$ & 51.9 & \\
\hline & Moderate or high & $/ / / / / / / / / /$ & 48.1 & \\
\hline
\end{tabular}

the proportion of overweight/obese children was found to be higher in low-SES families (32.2\%), in comparison to medium-SES (23.2\%) or high-SES (22.2\%) families. The proportion of overweight/obese children in foodinsecure households (52.5\%) was more than double the proportion in food-secure households (23.1\%). A higher proportion of overweight/obesity was also observed in children from single-parent families and children who consumed vegetables less than once a day, in comparison to children living in other types of families and children who consumed vegetables more frequently per day, respectively (see also adjusted odds ratios in Table 3 ). While these trends were similar in boys and girls, the statistically significant findings indicated in Table 2 appear to be driven by differences observed among girls; no statistically significant differences were observed among boys.

In Jamaica, childhood overweight/obesity was significantly associated only with family SES and household food insecurity. The proportion of overweight/obese children was higher in high-SES families (13\%) in comparison to medium-SES (12.2\%) or low-SES (6.8\%) families. Similarly, a higher proportion of overweight/ obesity was found in children from food-secure households $(12.1 \%)$ in comparison to those in food-insecure households (7.9\%).

Sex-specific analyses yielded several significant associations in both the Québec and Jamaican samples. For girls in Québec, childhood overweight/obesity was significantly associated with family SES, household food insecurity, family type, daily consumption of pastries, and daily consumption of vegetables. For boys, no association was observed between childhood overweight/obesity and any of the child, family, or household variables examined. In Jamaica, when the data were analyzed separately for girls and boys, only level of physical activity showed a significant association with overweight/obesity in girls, and only family SES was associated with overweight/obesity in boys.

Multivariate analyses (Table 3) revealed that children in food-insecure households in Québec had odds of 3.03 (95\% CI 1.8-5.0) for being overweight or obese, with odds of 4.99 (95\% CI 2.4-10.5) for being overweight or obese specifically in girls (when analyzed by gender) in comparison to children in food-secure households. Single-parenting also increased the odds of being 
Table 2 Frequency of overweight or obesity in Québec and Jamaican children by selected characteristics, by sex and by both sexes combined

\begin{tabular}{|c|c|c|c|c|c|c|c|}
\hline \multirow[b]{2}{*}{ Characteristic } & \multirow[b]{2}{*}{ Category } & \multicolumn{3}{|c|}{$\begin{array}{l}\text { Overweight or obese Québec } \\
\text { children }\end{array}$} & \multicolumn{3}{|c|}{$\begin{array}{l}\text { Overweight or obese Jamaican } \\
\text { children }\end{array}$} \\
\hline & & Girls & Boys & All & Girls & Boys & All \\
\hline \multirow[t]{2}{*}{ Sex } & Girl & $/ / / / / / / / / / / / /$ & $/ / / / / / / / / / / /$ & 26.3 & $/ / / / / / / / / / / /$ & $/ / / / / / / / / / / /$ & 12.6 \\
\hline & Boy & $/ / / / / / / / / / / / /$ & $/ / / / / / / / / / / / /$ & 25.3 & $/ / / / / / / / / / / / /$ & $/ / / / / / / / / / / / /$ & 9.2 \\
\hline \multirow[t]{3}{*}{ Family SES } & Tertile 1 (Low) & $34.5^{*}$ & 29.6 & $32.2^{*}$ & 8.3 & $5.3^{*}$ & $6.8^{*}$ \\
\hline & Tertile 2 (Medium) & 22.8 & 23.6 & 23.2 & 14.9 & 9.5 & 12.2 \\
\hline & Tertile 3 (High) & 21.6 & 23.0 & 22.2 & 14.2 & 11.6 & 13.0 \\
\hline \multirow[t]{2}{*}{ Household food insecurity } & No & $23.2^{*}$ & 23.1 & $23.1^{*}$ & 13.7 & 10.5 & $12.1^{*}$ \\
\hline & Yes & 66.0 & 34.6 & 52.5 & 9.8 & 6.1 & 7.9 \\
\hline \multirow[t]{3}{*}{ Family type } & Two-parent family & $21.7^{*}$ & 24.5 & $23.1^{*}$ & 13.8 & 10.8 & 12.5 \\
\hline & Blended family & 28.8 & 27.0 & 28.1 & 13.5 & 8.7 & 11 \\
\hline & Single-parent family & 41.9 & 27.6 & 35.2 & 10.8 & 8.2 & 9.5 \\
\hline \multirow[t]{3}{*}{ Pastry consumption } & Less than once a day & $30.7^{*}$ & 25.6 & 28.4 & 13.5 & 10 & 11.9 \\
\hline & Once to less than twice a day & 26.1 & 20.5 & 23.1 & 10.3 & 8.7 & 9.5 \\
\hline & Twice a day or more & 10.4 & 24.9 & 17.1 & 9.2 & 6.7 & 7.9 \\
\hline \multirow[t]{3}{*}{ Fruit consumption } & Less than once a day & 34.2 & 28.4 & 31.4 & 14.5 & 9.3 & 12 \\
\hline & Once to less than twice a day & 26.9 & 19.1 & 22.8 & 10.9 & 9.9 & 10.4 \\
\hline & Twice a day or more & 24.4 & 24.4 & 24.4 & 11.5 & 7.7 & 9.6 \\
\hline \multirow[t]{3}{*}{ Vegetable consumption } & Less than once a day & $41.9^{*}$ & 31.7 & $37.3^{*}$ & 13.1 & 9.1 & 11.2 \\
\hline & Once to less than twice a day & 19.9 & 22.7 & 21.2 & 12.5 & 9.4 & 10.9 \\
\hline & Twice a day or more & 21.9 & 21.4 & 21.6 & 12.5 & 9.6 & 11.1 \\
\hline \multirow[t]{2}{*}{ Physical activity (Québec) } & Same, lower or much lower than other children & 28.8 & 26.3 & 27.6 & $/ / / / / / / / / /$ & $/ / / / / / / / / /$ & $\mid / / / / / / / / /$ \\
\hline & Higher or much higher than other children & 20.4 & 23.4 & 21.8 & $/ / / / / / / / / /$ & $/ / / / / / / / / /$ & I/I/I/I/I/ \\
\hline \multirow[t]{2}{*}{ Physical activity (Jamaica) } & None or low & $/ / / / / / / / / /$ & $/ / / / / / / / / /$ & $/ / / / / / / / / /$ & $15.0^{*}$ & 9.1 & 12.5 \\
\hline & Moderate or high & $/ / / / / / / / / /$ & $/ / / / / / / / / /$ & $/ / / / / / / / / /$ & 10.1 & 9.2 & 9.6 \\
\hline
\end{tabular}

* Statistically significant association between the characteristic and the dependent variables within column (chi-square $\mathrm{P} \leq 0.05$ ).

overweight or obese by 63\% in Québec children. Family SES was no longer significant in multivariate analyses. In Jamaica, the association with childhood overweight/ obesity was reversed: higher SES was associated with increased overweight/obesity, and food insecurity was associated with reduced overweight/obesity. More specifically, Jamaican children in food-insecure households had odds of 0.65 (95\% CI 0.4-0.9) for being overweight or obese in comparison to those in food-secure households. Similarly, Jamaican children in middle SES (OR 1.83, 95\% CI 1.1-3.0) and high SES (OR 2.00, 95\% CI 1.3-3.2) families had increased odds of being

Table 3 Adjusted odds ratios (OR and $95 \% \mathrm{Cl}$ ) for overweight and obese Québec and Jamaican children by selected characteristics, by sex and by both sexes combined

\begin{tabular}{|c|c|c|c|c|c|c|c|}
\hline \multirow[b]{2}{*}{ Characteristic } & \multirow[b]{2}{*}{ Category } & \multicolumn{3}{|c|}{ Overweight or obese Québec children ${ }^{\#}$} & \multicolumn{3}{|c|}{ Overweight or obese Jamaican children ${ }^{\# \#}$} \\
\hline & & Girls & Boys & All & Girls & Boys & All \\
\hline \multirow[t]{3}{*}{ Family SES } & Tertile 1 (Low) & 1 & 1 & 1 & 1 & 1 & 1 \\
\hline & Tertile 2 (Middle) & $0.87(0.5-1.5)$ & $0.92(0.5-1.6)$ & $0.89(0.6-1.3)$ & $1.87(1.0-3.4)^{*}$ & $1.75(0.8-3.9)$ & $1.83(1.1-3.0)^{*}$ \\
\hline & Tertile 3 (High) & $0.97(0.5-1.7)$ & $0.91(0.5-1.7)$ & $0.95(0.6-1.4)$ & $1.74(0.9-3.3)$ & $2.37(1.2-4.9)^{*}$ & $2.00(1.3-3.2)^{*}$ \\
\hline \multirow[t]{3}{*}{ Family type } & Two-parent family & 1 & 1 & 1 & 1 & 1 & 1 \\
\hline & Blended family & $0.91(0.5-1.7)$ & $1.14(0.6-2.2)$ & $1.0(0.7-1.6)$ & $1.0(0.6-1.6)$ & $0.59(0.3-1.6)$ & $0.82(0.6-1.2)$ \\
\hline & Single-parent family & $1.82(0.9-3.4)$ & $1.37(0.7-2.5)$ & $1.63(1.0-2.5)^{*}$ & $0.79(0.4-1.3)$ & $0.69(0.3-1.2)$ & $0.74(0.5-1.1)$ \\
\hline \multirow[t]{2}{*}{ Household food insecurity } & No & 1 & 1 & 1 & 1 & 1 & 1 \\
\hline & Yes & $4.99(2.4-10.5)^{*}$ & $1.56(0.7-3.3)$ & $3.03(1.8-5.0)^{*}$ & $0.72(0.4-1.2)$ & $0.58(0.3-1.3)$ & $0.65(0.4-0.9)^{*}$ \\
\hline
\end{tabular}

*Significantly different from the reference category $(P \leq 0.05)$.

\# Models adjusted for consumption of pastry and vegetables and for physical activity.

\#\# Models adjusted for physical activity. 
overweight or obese in comparison to children in low SES families. When boys and girls were considered separately, only SES remained positively associated with overweight/obesity. In comparison to boys and girls in low SES families, boys from high-SES families had odds of 2.37 (95\% CI 1.2-4.9) for being overweight or obese, and girls from middle-SES families had odds of 1.87 (95\% CI 1.0-3.4). Odds ratio point estimates for boys from middle-SES families and girls from high-SES families increased but failed to reach statistical significance.

\section{Discussion}

The aims of this study were twofold. First, we sought to determine whether household food insecurity was significantly related to childhood overweight/obesity in Québec and in Jamaica. Second, we explored possible gender differences in these associations.

\section{Overweight and obesity}

Overall we found that the prevalence of overweight/obesity differed considerably between Québec (26\%) and Jamaica (11\%). Findings from the Québec sample corresponded to estimates for Canada as a whole [28] and were also comparable to estimates from other developed countries, such as the United States [52]. However, the prevalence of overweight/obesity among Jamaican children appeared lower than previously reported for similarly aged children [29]. The estimates obtained in the aforementioned study was, however, confined to the urban area of Kingston; whereas, data for our study were representative of all 14 parishes in Jamaica. The use of universal cut-points for overweight/obesity in both adults and children is somewhat controversial due to differences in race/ethnicity and the effects of maturation and genetics [53-55]. Our findings showed clearly that obesity is a critical health problem in both countries. The large disparity in the prevalence of overweight/obesity observed may be attributable to genetic, psychosocial, economic, dietary, and other environmental or behavioral factors, such as physical activity [53]. The disparity may also reflect differences in how the economies of these two countries are integrated into the global economy. In rural areas of developing countries, individuals may have less access to low-nutrient, energydense products that are high in sugar and fat, and indeed underweight can be more prevalent than overweight/obesity $[14,56]$.

\section{Household food insecurity}

The prevalence of food insecurity among children in Québec (9.4\%) was slightly higher than the one reported for the province of Québec through the 2008 Canadian Community Health Survey [30,57]. In 2008, 14.6\% of households in the United States reported food insecurity; however, it was not clear how many of these households included children [58]. The present study estimates household food insecurity among Jamaican children to be at a prevalence of $26.4 \%$; to our knowledge, these findings are the first to provide such an estimate. This percentage is disproportionately lower than estimates reported for other developing countries, including an urban population in Caracas, Venezuela (64\%) [59], urban and rural samples from Bolivia (70\%) [60], and school children 5-12 years old in Bogota, Columbia (76\%) [61].

\section{Association between household food insecurity and overweight/obesity}

The present report confirms our hypothesis indicating that in Québec, children who live in food-insecure households have higher odds of being overweight or obese in comparison to children who live in food-secure households. By contrast, in Jamaica food insecurity was associated with decreased odds of being overweight or obese. Similarly, recent studies in Canada and the United States have reported a positive association between food insecurity and childhood obesity [20-22]. However, other studies conducted in similar contexts (e.g., the United States and Mexico) reported negative [23-25] or no relationships between food insecurity and childhood obesity $[26,27,62]$. As Gundersen and colleagues (2008) suggested, these inconsistencies may be explained by differences in the way food insecurity was measured. For example, in a developing country like Trinidad, Gulliford (2003) showed that food insecurity was associated with an increased likelihood of underweight and not obesity among adults [63]. Unfortunately, studies that might shed light on the relationship between food insecurity and overweight/obesity in children are scarce in developing countries.

One possible explanation for disparities in the relationship between food insecurity and overweight/obesity between developed and developing middle-income countries is that the food-insecurity and overweight/ obesity pathways may be different. For example, in developed countries like Canada, social support services available to low-income and food-insecure families (e.g., food subsidies, food stamps, social assistance) have been associated with increased overweight/obesity $[64,65]$. This may be due to an increased consumption of cheaper and refined carbohydrates, sweetened beverages, high-fat meats, and lower consumption of fruits and vegetables because of monetary constraints. It is known that poorer families may substitute higher-quality foods for cheaper, lower-quality foods and this practice has been associated with overweight and obesity in developed countries [66-68]. 
By contrast, developing countries like Jamaica have limited social support systems to help with food subsidies. As a result, children from food-insecure and poorer families consume fewer total calories and so are less likely to be overweight or obese. Moreover, the present study's findings may explain why food insecurity and SES share a pathway in relation to overweight and obesity in both countries. Unlike the association between SES and overweight/obesity in Jamaica, the prevalence of overweight/obesity was higher among children from low-SES families and/or children from single-parent families in Québec. In multivariate analyses, SES did not prove to be a significant factor, probably due to the inadequate sample size and its attendant inability to show statistical significance. By contrast, in Jamaica, both variables remained significant, thereby associating high SES with overweight/obesity, whereas food insecurity was associated with reduced odds of overweight/ obesity.

\section{Gender differences}

In Québec, when boys and girls were considered separately, food insecurity significantly associated with being overweight or obese only in girls. In children from foodinsecure households in Québec, disproportionately more girls were overweight or obese than boys. Additionally, among children who were overweight, more girls than boys $(41.9 \%$ vs. $27.6 \%)$ had single-parent families, and more girls than boys came from low-SES families (34.5\% vs. $29.6 \%$ ). These findings show that the majority of girls and boys from food-insecure households lived in two very different family environments. This difference may explain the gender-specific relationship between household food insecurity and childhood overweight/obesity observed in our study. Moreover, given that, in almost all cases (99\%), the head of single-parent families were women in the Québec sample [69], perhaps having an overweight/obese mother exerted a strong influence on girls' body weights since modeling effectively transmits values, beliefs, and practices [70]; however this explanation requires further investigation. When the time and desire to cook are limited among single mothers, they may resort to buying inexpensive, energy-dense, processed foods that have been associated with the obesity epidemic [67].

On the other hand, in Jamaica the prevalence of overweight/obesity was higher among children living in two-parent and blended families, although group differences were not significant at the univariate or multivariate levels. Additionally, boys from higher SES families had greater odds of being overweight/obese than did girls. A positive association between higher SES and overweight/obesity among men, women, and children has been reported in the literature on developing countries [16,17]. Among Jamaican children, the food insecurity-overweight/obesity relationship yielded similar results when boys and girls were analyzed separately. This result has been previously reported for adults in other developing countries [63].

The gender-based differences observed in the present study among children from Québec have been previously reported for adults in other developed countries, such that women in food-insecure households were found to have a higher prevalence of overweight/obesity in comparison to men $[45,65,71]$. Few studies have examined gender differences in the association between food insecurity and overweight/obesity in children. Two studies found that food-insecure girls were more likely to be overweight than their food-secure counterparts; whereas, among boys, there were no significant findings $[22,41]$. In developing countries like Jamaica, these previously unstudied emergent differences may well be attributable to the status of the nutritional and epidemiological transition [72,73]. Other mitigating factors, such as family type and SES which our study shows to be present in both developed and developing countries, are consistent with those reported in the literature $[16,17,64]$.

\section{Strengths and limitations}

The most notable strength of this study is that both samples were statistically representative of their populations and both employed standardized weights. The response rate was greater than or equal to $85 \%$ in both samples. The Jamaican sample size was adjusted for a $10 \%$ refusal rate. The results of the study can thus be generalized to other cases. In addition, objective anthropometric measures of body mass index were obtained.

As our study explores the relationship between food insecurity and overweight/obesity in children from both a developed and a developing country, certain limitations exist. First, given the cross-sectional nature of the data, causality cannot be assigned to the associations observed in this study. Elucidating these relationships will require retrospective longitudinal studies that make it possible to examine temporal sequences of events [41]. Issues relating to the measurement of food insecurity also did not allow us to examine the effects of different levels of food insecurity on overweight/obesity. Furthermore, in the Québec sample, children's level of physical activity was parent-reported rather than measured; thus, children were classified according to subjective information. A number of studies have shown that parents have difficulty accurately estimating and recalling their children's physical activity patterns (e.g., type, intensity, duration, frequency) [34]. Finally, the derivation of the SES variable in the Jamaican sample did not include conventional indicators, such as income and 
education, as they were not available. This may have resulted in a SES index with reduced discriminatory capacity.

\section{Conclusions}

Food insecurity appears to be positively associated with childhood overweight/obesity in children from the province of Québec, Canada. An inverse relationship is observed among children in Jamaica, a developing country. Gender differences are also apparent in the food insecurity-childhood overweight/obesity association in Québec, such that significantly more girls are found to be "at risk" in comparison to boys. Sex differences in the association between SES, family type, and childhood overweight/obesity are observed in both Québec and Jamaica. The findings of this study suggest that public health interventions which aim to stem the epidemic of overweight/obesity should look beyond biology and individual behaviours to the root causes of the epidemic. Family dynamics, gender differences, and trends in the global food system all influence the spread of overweight/obesity, both in developed and developing countries.

\section{Abbreviations \\ SES: socioeconomic status; QLSCD: Québec Longitudinal Study of Child Development; BMI: Body Mass Index; CDC: Centers for Disease Control and Prevention; FAO: Food and Agriculture Organization; USDA: United States Department of Agriculture; FFQ: Food Frequency Questionnaire; IPAQ: International Physical Activity Questionnaire; STATIN: Statistical Institute of Jamaica; OR: Odds Ratios}

\section{Acknowledgements}

The Québec portion of this study has been partly financed by the Canadian Institute of Health Research. The analyses were performed using data from the Québec Longitudinal Study of Child Development (1998-2010) (QLSCD), conducted by Santé Québec, a division of the Institut de la Statistique du Québec (ISQ) and funded by the Ministry of Health and Social Services of Québec. In Jamaica, the analyses were performed using data from the Jamaica Youth Risk and Resiliency Behaviour Survey 2005, conducted by the Sir Arthur Lewis Institute of Social and Economic Studies with funding from the USAID and Ministry of Health Jamaica. Technical assistance was provided by MEASURE Evaluation, Tulane University.

\section{Author details}

${ }^{1}$ Faculty of Medicine, University of Ottawa, Institute of Population Health, 1 Stewart Street, office 303, Ottawa, Ontario, K1N 6N5, Canada. ${ }^{2}$ Epidemiology Research Unit- TMRI, University of the West Indies, Mona, Kingston 7, Jamaica. ${ }^{3}$ Institute of Population Health, University of Ottawa, 1 Stewart Street, Office 304, Ottawa, Ontario, K1N 6N5, Canada. ${ }^{4}$ Faculty of Medical Sciences, Deans Office, University of the West Indies, Mona, Kingston 7, Jamaica. ${ }^{5}$ Sir Arthur Lewis Institute of Social Sciences and Economic Studies, University of the West Indies, Mona, Kingston 7, Jamaica.

\section{Authors' contributions}

LD is the principal investigator and was primarily responsible for the conceptualization of the study. DF, DB, and MG analyzed the data, and DF, $D B$, and FT wrote the manuscript. GGS, KF, and RW critically revised the manuscript for important intellectual content. All authors read and approved the final manuscript.

\section{Competing interests}

The authors declare that they have no competing interests.
Received: 17 November 2010 Accepted: 31 March 2011

Published: 31 March 2011

\section{References}

1. Wang $Y$, Lobstein T: Worldwide trends in childhood overweight and obesity. Int J Pediatr Obes 2006, 1:11-25.

2. Rokholm B, Baker $J L$, Sorensen TIA: The levelling off of the obesity epidemic since the year 1999 - a review of evidence and perspectives. Obes Rev 2010, 11:835-846.

3. Ball GDC, McCargar LJ: Childhood obesity in Canada: A review of prevalence estimates and risk factors for cardiovascular diseases and type 2 diabetes. Can J Appl Physiol 2003, 28:117-140.

4. Daniels SR: The consequences of childhood overweight and obesity. Future Child 2006, 16:47-67.

5. Reilly JJ, Methven E, McDowell ZC, Hacking B, Alexander D, Stewart L, Kelnar CJH: Health consequences of obesity. Arch Dis Child 2003, 88:748-752.

6. Slyper AH: The pediatric obesity epidemic: Causes and controversies. J Clin Endocrinol Metab 2004, 89:2540-2547.

7. Francis DK, Van den Broeck J, Younger N, McFarlane S, Rudder K, GordonStrachan G, Grant A, Johnson A, Tulloch-Reid M, Wilks R: Fast-food and sweetened beverage consumption: association with overweight and high waist circumference in adolescents. Public Health Nutr 2009, 12:1106-1114.

8. Harnack LJ, Jeffery RW, Boutelle KN: Temporal trends in energy intake in the United States: an ecologic perspective. Am J Clin Nutr 2000, 71:1478-1484.

9. Kant AK, Graubard BI: Energy density of diets reported by American adults: association with food group intake, nutrient intake, and body weight. Int J Obes 2005, 29:950-956.

10. Nicklas TA, Elkasabany A, Srinivasan SR, Berenson G: Trends in nutrient intake of 10-year-old children over two decades (1973-1994) The Bogalusa Heart Study. Am J Epidemiol 2001, 153:969-977.

11. Troiano RP, Briefel RR, Carroll MD, Bialostosky K: Energy and fat intakes of children and adolescents in the United States: data from the National Health and Nutrition Examination Surveys. Am J Clin Nutr 2000, 72:1343S-1353S.

12. Doak CM, Adair LS, Bentley M, Monteiro C, Popkin BM: The dual burden household and the nutrition transition paradox. Int J Obes 2005, 29:129-136.

13. Wang YF, Monteiro C, Popkin BM: Trends of obesity and underweight in older children and adolescents in the United States, Brazil, China, and Russia. Am J Clin Nutr 2002, 75:971-977.

14. Kosti Rl, Panagiotakos DB: The epidemic of obesity in children and adolescents in the world. Cent Eur J Public Health 2006, 14:151-159.

15. Shrewsbury $\vee$, Wardle J: Socioeconomic status and adiposity in childhood: A systematic review of cross-sectional studies 1990-2005. Obesity (Silver Spring) 2008, 16:275-284.

16. McLaren L: Socioeconomic status and obesity. Epidemiol Rev 2007, 29:29-48.

17. Sobal J, Stunkard AJ: Socioeconomic-Status and Obesity - A Review of the Literature. Psychol Bull 1989, 105:260-275.

18. Mendez MA, Monteiro CA, Popkin BM: Overweight exceeds underweight among women in most developing countries. Am J Clin Nutr 2005, 81:714-721.

19. Food and Agriculture Organization (FAO): Food Security. Rome: FAO; 2006.

20. Casey PH, Simpson PM, Gossett JM, Bogle ML, Champagne CM, Connell C, Harsha D, McCabe-Sellers B, Robbins JM, Stuff JE: The association of child and household food insecurity with childhood overweight status. Pediatrics 2006, 118:E1406-E1413.

21. Dubois L, Farmer A, Girard M, Porcherie M: Family food insufficiency is related to overweight among preschoolers. Soc Sci Med 2006, 63:1503-1516.

22. Jyoti DF, Frongillo EA, Jones SJ: Food insecurity affects school children's academic performance, weight gain, and social skills. J Nutr 2005, 135:2831-2839.

23. Jimenez-Cruz A, Bacardi-Gascon M, Spindler AA: Obesity and hunger among Mexican-Indian migrant children on the US-Mexico border. Int $J$ Obes 2003, 27:740-747

24. Matheson DM, Varady J, Varady A, Killen JD: Household food security and nutritional status of Hispanic children in the fifth grade. Am J Clin Nutr 2002, 76:210-217. 
25. Rose D, Bodor JN: Household food insecurity and overweight status in young school children: Results from the Early Childhood Longitudinal Study. Pediatrics 2006, 117:464-473.

26. Gundersen C, Lohman BJ, Eisenmann JC, Garasky S, Stewart SD: Childspecific food insecurity and overweight are not associated in a sample of 10- to 15-year-old low-income youth. J Nutr 2008, 138:371-378.

27. Kaiser LL, Melgar-Quinonez HR, Lamp CL, John MC, Sutherlin JM, Harwood JO: Food security and nutritional outcomes of preschool-age Mexican-American children. J Am Diet Assoc 2002, 102:924-929.

28. Shields M: Measured obesity: overweight Canadian children and adolescents in Nutrition: Findings from the Canadian Community Health survey. Ottawa: Statistics Canada; 2009.

29. Jackson M, Samms-Vaughan M, Ashley D: Nutritional status of 11-12-yearold Jamaican children: coexistence of under- and overnutrition in early adolescence. Public Health Nutr 2002, 5:281-288.

30. Canada Health: Canadian Community Health Survey. Ottawa: Health Canada; 2008.

31. Dubois L, Bédard B, Girard M, Beauchesne E: L'alimentation. Etude longitudinale du développement des enfants du Québec (ELDEQ 19982002). Les nourrissons de 5 mois. Québec: Institut de la statistique du Québec 2000.

32. Fox K, Gordon-Strachan G: Jamaican Youth Risk and Resiliency Behaviour Survey 2005. 2007.

33. Planning Institute of Jamaica: Planning Institute of Jamaica, Economic and Social Survey of Jamaica. Planning Institute of Jamaica: Kingston; 2004.

34. Goran Ml: Measurement issues related to studies of childhood obesity: Assessment of body composition, body fat distribution, physical activity, and food intake. Pediatrics 1998, 101:505-518.

35. Statistical Institute of Jamaica (STATIN): Demographic Statistics (2001). Kingston: STATIN; 2001.

36. global Tobacco Surveillance System Collaborating Group: Global Tobacco Surveillance System (GTSS): purpose, production, and potential. J Sch Health 2005, 75:15-24.

37. Desrosiers $H$, Dubois $L$, Beal Bédard: Enquête de nutrition auprès des enfants québecois de 4 ans. Québec: Institut de la Statistique du Québec 2005.

38. Cole TJ, Bellizzi MC, Flegal KM, Dietz WH: Establishing a standard definition for child overweight and obesity worldwide: international survey. Br Med J 2000, 320:1240-1243.

39. Canada Health: Canadian Community Health Survey Cycle 2.2, Nutrition Income-Related Household Food Security in Canada. Ottawa: Health Canada; 2004.

40. Anderson SA: Core Indicators of Nutritional State for Difficult-To-Sample Populations. J Nutr 1990, 120:1559-1599.

41. Alaimo K, Olson CM, Frongillo EA: Low family income and food insufficiency in relation to overweight in US children - Is there a paradox? Arch Pediatr Adolesc Med 2001, 155:1161-1167.

42. Rose D, Gundersen C, Oliveira V: Socio-economic determinant of food insecurity in the United States: Evidence from the SIPP and CSF II datasets. Washington, DC: US Department of Agriculture; 1998, ERS-TB-1869 edn.

43. Briefel RR, Woteki CE: Development of Food Sufficiency Questions for the 3Rd National-Health and Nutrition Examination Survey. J Nutr Educ 1992, 24:S24-S28.

44. Carlson S, Briefel RR: The USDA and NHANES food insufficiency question as an indicator of hunger and food insecurity. In Conference on food security measurement and research: Papers and proceedings. Alexandria, VA: US Department of Agriculture, Food and Consumer Services; 1995.

45. Basiotis PP: Validity of the self-reported food insufficiency status item in the US Department of Agriculture Food Consumption Surveys. In Proceedings of the 1992 annual meeting of the American council in the consumer interest, March 25-28. Toronto, ON; 1992.

46. Cristofar SP, Basiotis PP: Dietary Intakes and Selected Characteristics of Women Ages 19-50 Years and Their Children Ages 1-5 Years by Reported Perception of Food Sufficiency. J Nutr Educ 1992, 24:53-58.

47. Pan American Health Organization: The Caribbean Youth Survey 1997. 1997.

48. Craig CL, Marshall AL, Sjostrom M, Bauman AE, Booth ML, Ainsworth BE, Pratt M, Ekelund U, Yngve A, Sallis JF, Oja P: International physical activity questionnaire: 12-country reliability and validity. Med Sci Sports and Exerc 2003, 35:1381-1395.
49. Jetté M, Des Groseilliers L: Survey description and methodology in Longitudinal Study of Child Development in Québec (ELDEQ 1998-2002) (Vol. 1, 1). Québec: Institut de la statistique du Québec; 2000.

50. Willms JD, Shields M: A measure of socioeconomic status for the National Longitudinal Study of Children. Report prepared for Statistics Canada 1996.

51. Cox B, Cohen S: Methodological Issues jor Health Care Surveys. New York: Dekker; 1985.

52. Hedley AA, Ogden CL, Johnson CL, Carroll MD, Curtin LR, Flegal KM: Prevalence of overweight and obesity among US children, adolescents, and adults, 1999-2002. J Am Med Assoc 2004, 291:2847-2850.

53. Bray GA: In defense of a body mass index of 25 as the cut-off point for defining overweight. Obes Res 1998, 6:461-462.

54. Deurenberg $P$, Yap $M$, van Staveren WA: Body mass index and percent body fat: a metaanalysis among different ethnic groups. Int J Obes 1998, 22:1164-1171.

55. World Health Organization: The Asia-Pacific Perspective: Redefining Obesity and its Treatment. Sydney: Health Communications; 2000.

56. Sobal J: Commentary: Globalization and the epidemiology of obesity. Int J Epidemiol 2001, 30:1136-1137.

57. Health Canada: Canadian Community Health Survey Cycle 2.2 Nutrition Income-Related Household Food Security in Canada. Ottawa: Health Canada; 2004.

58. Nord M, Andrews M, Carlson S: Household Food Security in the United States, 2008. 2009.

59. Lorenzana PA, Mercado C: Measuring household food security in poor Venezuelan households. Public Health Nutr 2002, 5:851-857.

60. Melgar-Quinonez HR, Zubieta AC, MkNelly B, Nteziyaremye A, Gerardo MFD, Dunford C: Household food insecurity and food expenditure in Bolivia, Burkina Faso, and the Philippines. J Nutr 2006, 136:1431-1437.

61. Isanaka S, Mora-Plazas M, Lopez-Arana S, Baylin A, Villamor E: Food insecurity is highly prevalent and predicts underweight but not overweight in adults and school children from Bogota, Colombia. J Nutr 2007, 137:2747-2755.

62. Gundersen C, Garasky S, Lohman BJ: Food Insecurity Is Not Associated with Childhood Obesity as Assessed Using Multiple Measures of Obesity. J Nutr 2009, 139:1173-1178.

63. Gulliford MC, Mahabir D, Rocke B: Food insecurity, food choices, and body mass index in adults: nutrition transition in Trinidad and Tobago. Int J Epidemiol 2003, 32:508-516.

64. Gibson LY, Byrne SM, Davis EA, Blair E, Jacoby P, Zubrick SR: The role of family and maternal factors in childhood obesity. Med J Aust 2007, 186:591-595.

65. Townsend MS, Peerson J, Love B, Achterberg C, Murphy SP: Food insecurity is positively related to overweight in women. J Nutr 2001, 131:1738-1745.

66. Dietz WH: Does Hunger Cause Obesity. Pediatrics 1995, 95:766-767.

67. Drewnowski A, Specter SE: Poverty and obesity: the role of energy density and energy costs. Am J Clin Nutr 2004, 79:6-16.

68. Wilde PE, Peterman JN: Individual weight change is associated with household food security status. J Nutr 2006, 136:1395-1400.

69. Institut de la Statistique du Québec: Family, child care and Neighbourhood characteristics. Québec; 2000.

70. Maccoby EE: Social Development. Psychological Growth and the ParentChild Relationship New York: Harcourt Brace Janovich; 1980.

71. Olson CM: Nutrition and health outcomes associated with food insecurity and hunger. J Nutr 1999, 129:521S-524S.

72. Gulliford MC: Epidemiological transition in Trinidad and Tobago, West Indies 1953-1992. Int J Epidemiol 1996, 25:357-365.

73. Wilks R, Bennett F, Forrester T, McFarlane-Anderson N: Chronic diseases: the new epidemic. West Indian Med J 1998, 47(4):40-44.

\section{Pre-publication history}

The pre-publication history for this paper can be accessed here: http://www.biomedcentral.com/1471-2458/11/199/prepub

doi:10.1186/1471-2458-11-199

Cite this article as: Dubois et al:: Household food insecurity and childhood overweight in Jamaica and Québec: a gender-based analysis. BMC Public Health 2011 11:199. 